\title{
Positive Almost Periodic Solution for a Model of Hematopoiesis with Infinite Time Delays and a Nonlinear Harvesting Term
}

\author{
Hui Zhou, ${ }^{1}$ Wen Wang, ${ }^{1}$ and Zongfu Zhou ${ }^{2}$ \\ ${ }^{1}$ Department of Mathematics, Hefei Normal University, Hefei 230601, China \\ ${ }^{2}$ School of Mathematical Science, Anhui University, Hefei 230039, China \\ Correspondence should be addressed to Hui Zhou; zhouhui0309@126.com
}

Received 4 June 2013; Accepted 29 August 2013

Academic Editor: Peixuan Weng

Copyright (c) 2013 Hui Zhou et al. This is an open access article distributed under the Creative Commons Attribution License, which permits unrestricted use, distribution, and reproduction in any medium, provided the original work is properly cited.

\begin{abstract}
A generalized model of Hematopoiesis with infinite time delays and a nonlinear harvesting term is investigated. By utilizing a fixed point theorem of the differential equations and constructing a suitable Lyapunov functional, we establish some conditions which guarantee the existence of a unique positive almost periodic solution and the exponential convergence of the system. Finally, we give an example to illustrate the effectiveness of our results.
\end{abstract}

\section{Introduction}

Recently, the existence problem of periodic solutions or almost periodic solutions has been one of the most attracting topics in the qualitative theory of functional differential equations because it is applied extensively in the physical sciences. Neutral functional differential equations arise in many areas of applied mathematics; it has received considerable attention in the recent decades. There have been many remarkable works (see, [1-5]). It is well known that there has been considerable interest in the existence of almost periodic solutions of functional differential equations [3-9].

There are many works about Hematopoiesis model due to its real applications [10-17]. Wang and Zhang [16] proved the existence, nonexistence, and uniqueness of positive almost periodic solution of the model of Hematopoiesis:

$$
x^{\prime}(t)=-a(t) x(t)+\frac{b(t)}{1+x^{n}(t-\tau(t))}, \quad n>1,
$$

which was used by Mackey and Glass [18] as a model for the dynamics of Hematopoiesis ( blood cells production). On one hand, as pointed out in [19], in the real world, the delays in differential equations of populations, ecology, and dynamic problems are usually infinite time delays; for example, Zhou et al. [20] studied positive almost periodic solutions for a class of Lasota-Wazewska model with infinite time delays; On the other hand, assuming that a harvesting function is a function of the delayed estimate of the true ecological and dynamic models, Zhou et al. [21] presented an overview of the results on the classical Nicholsons proposed Nicholsons, blowflies model with a linear harvesting term. The investigation of biological dynamics with harvesting is an important subject in the exploitation of biological resources which is related to the optimal management of renewable resources [21, 22].

Motivated by the above, the main aim of this paper is to obtain the conditions for the existence and exponential convergence of the positive almost solutions for the following model of Hematopoiesis with infinite time delays and a nonlinear harvesting term:

$$
\begin{aligned}
x^{\prime}(t)= & -a(t) x(t)+\sum_{i=1}^{m} b_{i}(t) \int_{0}^{+\infty} \frac{k_{i}(s)}{1+x^{n}(t-s)} d s \\
& -H(t, x(t-\sigma(t))), \quad t, \sigma \in R, n>1 .
\end{aligned}
$$

Under proper assumptions, we will obtain a unique positive almost periodic solution of (2) by using the method of a fixed point theorem. A model with infinite time delays and nonlinear harvesting terms is more realistic than that with discrete time delays and linear harvesting term. To our best knowledge, there are some works about Hematopoiesis model with discrete delays and a linear harvesting term [2123]. Apparently, our results are more meaningful in reality, 
so it is worthwhile continuing to study the existence and convergence of positive almost periodic solutions of (2).

For convenience, we will introduce some notations:

$$
\begin{aligned}
& h^{+}=\sup _{t \in R} h(t), \quad h^{-}=\inf _{t \in R} h(t), \\
& f^{+}=\sup _{(t, x) \in\left(R, R^{n}\right)} f(t, x), \quad f^{-}=\inf _{(t, x) \in\left(R, R^{n}\right)} f(t, x),
\end{aligned}
$$

where $h(t)$ and $f(t, x)$ are bounded continuous and uniformly bounded continuous functions, respectively. The following conditions (H1) hold:

$$
a^{-}>0, \quad b_{i}^{-}>0, \quad H^{-}>0, \quad i=1,2, \ldots, m .
$$

In (2), throughout this paper, the following hypotheses conditions hold:

(H2) $a$ and $b_{i}(i=1,2, \ldots, m)$ are almost periodic; there exists a compact subset $S \subset R^{n}$ such that $\sigma(t) \subset S$ is almost;

(H3) $H: R \times R^{n} \rightarrow R_{+}$are uniformly almost periodic and there exists a constant $L_{H}>0$ such that

$$
\|H(t, x)-H(t, y)\| \leq L_{H}\|x-y\| ;
$$

(H4) the delay kernel $k_{i}(t) \in C\left([0,+\infty), R_{+}\right)$and $\int_{0}^{+\infty} k_{i}(t) d t=1, i=1,2, \ldots, m$.

Let $C=C\left([-r, 0], R_{+}\right)$be the bounded continuous functions space. $C$ is equipped with usual supremum norm $\|$. $\|$. If $x(t)$ is continuous and defined on $\left[-r+t_{0}, \sigma\right)$ with $t_{0}, \sigma \in R$, then, for all $t \in\left[t_{0}, \sigma\right)$, we define $x_{t}(\theta)=x(t+\theta)$ for all $\theta \in[-r, 0]$. In view of the actual applications of system (2), the admissible initial conditions $x_{t_{0}}=\varphi, \varphi \in C\left([-r, 0], R_{+}\right)$, and $\varphi(0)>0$ are needed. We write $x_{t}\left(t_{0}, \varphi\right)\left(x\left(t ; t_{0}, \varphi\right)\right)$ for an admissible solution of (2) with the above initial conditions. We let $\left[t_{0}, \eta(\varphi)\right)$ be the maximal rightinterval of existence of $x_{t}\left(t_{0}, \varphi\right)$.

The rest of the paper is organized as follows. In Section 2, we shall introduce some definitions and Lemmas which play important roles in the proofs of our main results. In Section 3, some conditions for the existence of positive almost periodic solutions and that all solutions converge exponentially to a positive almost solution of (2) are established. In Section 4, we give an example to illustrate the effectiveness of our results.

\section{Preliminaries}

Now let us state the following definitions and lemmas, which will be useful in proving our main results.

Definition 1 (see [9]). A function $f: R \rightarrow R^{n}$ is said to be almost periodic, if for any $\varepsilon>0$ there is a constant $l(\varepsilon)>0$ such that in any interval of length $l(\varepsilon)$ there exists $\tau$ such that the inequality

$$
\|f(t+\tau)-f(t)\|<\varepsilon
$$

is satisfied for all $t \in R$. The number $\tau$ is called an $\varepsilon$-translation number of $f(t)$.
Definition 2 (see [7, 9]). A function $f: R \times R^{n} \rightarrow R^{n}$ is said to be almost periodic in $t$ uniformly for $x \in R^{n}$, if for any $\varepsilon>0$ and for each compact subject $D$ of $R^{n}$ there is a constant $l(\varepsilon)>0$, in any interval of length $l(\varepsilon)$; there exists $\tau$ such that the inequality

$$
\|f(t+\tau, x)-f(t, x)\|<\varepsilon
$$

is satisfied for all $(t, x) \in R \times D$.

Lemma 3 (see [9]). If $F: R \times R^{n} \rightarrow R^{n}$ is an almost periodic, in $t$ uniformly for $x \in R^{n}, \phi(t)$ is almost periodic, and $\phi(t) \subset S$ for all $t \in R$, with $S$ being a compact subset of $R^{n}$, then $f(t, \phi(t))$ is almost periodic.

Definition 4 (see $[7,9]$ ). Let $x \in R^{n}$ and $C(t)$ be an $n \times x$ continuous matrix defined on $R$. The linear system

$$
x^{\prime}(t)=C(t) x
$$

is said to admit an exponential dichotomy on $R$ if there exist positive constants $\lambda, k$, a projection $P$, and the fundamental solution matrix $X(t)$ of (8) satisfying

$$
\begin{gathered}
\left\|X(t) P X^{-1}(s)\right\| \leq \lambda e^{-k(t-s)}, \quad t \geq s, \\
\left\|X(t)(I-P) X^{-1}(s)\right\| \leq \lambda e^{-k(s-t)}, \quad t \leq s .
\end{gathered}
$$

Lemma 5 (see $[7,9])$. Let $c_{i}(t)$ be an almost periodic function on $R$ and

$$
M\left[c_{i}\right]=\lim _{T \rightarrow+\infty} \frac{1}{T} \int_{t}^{t+T} c_{i}(s) d s>0, \quad i=1,2, \ldots, n .
$$

Then the linear system $\dot{x}(t)=C(t) x(t)$ admits an exponential dichotomy on $R^{n}$, where $C(t)=\operatorname{diag}\left(-c_{1}(t),-c_{2}(t), \ldots,-c_{n}(t)\right)$.

Lemma 6 (see [9]). If the linear system (8) admits an exponential dichotomy, then the almost periodic system

$$
x^{\prime}(t)=C(t) x+f(t, x)
$$

has a unique almost periodic solution $x(t)$, and

$$
\begin{aligned}
x(t)= & \int_{-\infty}^{t} X(t) P X^{-1}(s) f(s, x(s)) d s \\
& -\int_{t}^{\infty} X(t)(I-P) X^{-1}(s) f(s, x(s)) d s .
\end{aligned}
$$

\section{Main Results}

In this section, we state and prove our main results concerning the existence of positive almost solutions of (2). Let $B$ denote the set of almost periodic function on $R$. For any $\varphi \in B$, we define $\|\varphi\|_{B}:=\sup _{t \in R}|\varphi(t)|$; obviously, $B$ is a Banach space. 
For any $u \in B$, considering an auxiliary equation,

$$
\begin{aligned}
x^{\prime}(t)= & -a(t) x(t) \\
& +\sum_{i=1}^{m} b_{i}(t) \int_{0}^{+\infty} \frac{k_{i}(s)}{1+u^{n}(t-s)} d s \\
& -H(t, u(t-\sigma(t))) .
\end{aligned}
$$

Let us define the operator $\Phi$ by

$$
\begin{array}{rl}
(\Phi u)(t)=\int_{-\infty}^{t} & g(s) \\
\times & {\left[\sum_{i=1}^{m} b_{i}(s) \int_{0}^{+\infty} \frac{k_{i}(r)}{1+u^{n}(s-r)} d r\right.} \\
& \quad-H(s, u(s-\sigma(s)))] d s,
\end{array}
$$

where

$$
g(s)=e^{-\int_{s}^{t} a(r) d r} .
$$

It is clear that (2) has one unique positive almost periodic solution if and only if the operator $\Phi$ has exactly one fixed point. We set and suppose that

$$
\begin{gathered}
M_{1}=\frac{\sum_{i=1}^{m} b_{i}^{+}+H^{+}}{a^{-}}, \\
M_{2}=\frac{\sum_{i=1}^{m} b_{i}^{-} /\left(1+M_{1}^{n}\right)-H^{+}}{a^{+}}>0 .
\end{gathered}
$$

Lemma 7. If (H1) holds and $C^{0}:=\left\{\varphi \mid \varphi \in C, M_{2}<\varphi(t)<\right.$ $\left.M_{1}, t \in[-r, 0]\right\}$. Then, for $\varphi \in C^{0}$, the solution $x\left(t ; t_{0}, \varphi\right)$ of (2) satisfies $M_{2}<x\left(t ; t_{0}, \varphi\right)<M_{1}$, for all $t \in\left[t_{0}, \eta(\varphi)\right)$ and $\eta(\varphi)=+\infty$.

Proof. Set $x(t)=x\left(t ; t_{0}, \varphi\right)$. Let $[0, T) \subseteq\left[t_{0}, \eta(\varphi)\right)$ be an interval such that $0<x(t)$ for all $t \in\left[t_{0}, T\right)$. We prove

$$
0<x(t)<M_{1}, \quad \forall t \in\left[t_{0}, T\right) .
$$

By method of contradiction, if (17) does not hold, there exists $t_{1} \in\left(t_{0}, T\right)$ such that $x\left(t_{1}\right)=M_{1}$ and $0<x(t)<M_{1}$ for all $t \in\left[t_{0}-r, t_{1}\right)$. Calculating the derivative of $x(t)$ and by $(\mathrm{H} 1)$, we have that

$$
\begin{aligned}
0 & \leq x^{\prime}\left(t_{1}\right) \\
& =-a\left(t_{1}\right) x\left(t_{1}\right) \\
& +\sum_{i=1}^{m} b_{i}\left(t_{1}\right) \int_{0}^{+\infty} \frac{k_{i}(s)}{1+x^{n}\left(t_{1}-s\right)} d s \\
& -H\left(t_{1}, x\left(t_{1}-\sigma\left(t_{1}\right)\right)\right) \\
& \leq-a\left(t_{1}\right) x\left(t_{1}\right)+\sum_{i=1}^{m} b_{i}\left(t_{1}\right) \int_{0}^{+\infty} \frac{k_{i}(s)}{1+x^{n}\left(t_{1}-s\right)} d s \\
& \leq-a^{-} M_{1}+\sum_{i=1}^{m} b_{i}^{+}=-H^{+}<0,
\end{aligned}
$$

Next we prove that

$$
x(t)>M_{2}, \quad \forall t \in\left[t_{0}, \eta(\varphi)\right) .
$$

Otherwise, there exists $t_{2} \in\left(t_{0}, \eta(\varphi)\right)$ such that $x\left(t_{2}\right)=$ $M_{2}$ and $x(t)>M_{2}$ for all $t \in\left[t_{0}-r, t_{2}\right)$.

So from (17), we get $M_{2}<x(t)<M_{1}$, for $t \in\left[t_{0}-r, t_{2}\right)$. Calculating the derivative of $x(t)$, together with (H1) and (17), we obtain that

$$
\begin{aligned}
0 & \geq x^{\prime}\left(t_{2}\right) \\
& =-a\left(t_{2}\right) x\left(t_{2}\right) \\
& +\sum_{i=1}^{m} b_{i}\left(t_{2}\right) \int_{0}^{+\infty} \frac{k_{i}(s)}{1+x^{n}\left(t_{2}-s\right)} d s \\
& -H\left(t_{2}, x\left(t_{2}-\sigma\left(t_{2}\right)\right)\right) \\
& \geq-a\left(t_{2}\right) x\left(t_{2}\right)+\sum_{i=1}^{m} b_{i}\left(t_{2}\right) \int_{0}^{+\infty} \frac{k_{i}(s)}{1+x^{n}\left(t_{2}-s\right)} d s-H^{+} \\
& >-a^{+} M_{2}+\sum_{i=1}^{m} \frac{b_{i}^{-}}{1+M_{1}^{n}}-H^{+}=0,
\end{aligned}
$$

which is a contradiction and implies that (19) holds. From Theorem 2.3.1 in [24], we have that $\eta(\varphi)=+\infty$. The proof of Lemma 7 is completed.

Theorem 8. Assume that (H1) $-(H 4)$ and $L_{H}+n \sum_{i=1}^{m} b_{i}^{+} \leq a^{-}$. Then, there exists a unique positive almost periodic solution of (2) in the region $B^{*}:=\left\{\varphi \mid \varphi \in B, M_{2} \leq \varphi(t) \leq M_{1}\right.$, for all $t \in$ $R\}$.

Proof. For any $u \in B$, by (H2)-(H3) and Lemma 6, we know that (14) is an almost periodic differential equation. Since $\alpha^{-}>0$, notice that $M[\alpha]>0$. From Lemmas 3 and 5 , we obtain that the system (2) has exactly one almost periodic solution:

$$
\begin{aligned}
& u(t)=\int_{-\infty}^{t} g(s) \\
& \times\left[\sum_{i=1}^{m} b_{i}(s) \int_{0}^{+\infty} \frac{k_{i}(r)}{1+u^{n}(s-r)} d r\right. \\
&\quad-H(s, u(s-\sigma(s)))] d s .
\end{aligned}
$$

Define a mapping $T(u(t))=u(t)$, for all $u \in B$. 
It is easy to see that $B^{*}$ is a closed subset of $B$. For any $u \in B^{*}, t \in R$, from (H1) and (14), we obtain that

$$
\begin{aligned}
u(t) \leq & \int_{-\infty}^{t} g(s) \\
& \times\left[\sum_{i=1}^{m} b_{i}(s) \int_{0}^{+\infty} \frac{k_{i}(r)}{1+u^{n}(s-r)} d r\right. \\
& +H(s, u(s-\sigma(s)))] d s \\
\leq & \frac{\sum_{i=1}^{m} b_{i}^{+}+H^{+}}{a^{-}}=M_{1}, \\
u(t) \geq & \int_{-\infty}^{t} g(s)\left[\sum_{i=1}^{m} b_{i}(s) \int_{0}^{+\infty} \frac{k_{i}(r)}{1+u^{n}(s-r)} d r\right] d s-H^{+} \\
\geq & \frac{\sum_{i=1}^{m} b_{i}^{-} /\left(1+M_{1}^{n}\right)-H^{+}}{a^{+}}=M_{2} .
\end{aligned}
$$

Thus, the mapping $T$ is a self-mapping from $B^{*}$ to $B^{*}$. Next, we show that the mapping $T$ is a contraction mapping on $B^{*}$. Actually, for any $u, v \in B^{*}$, we have

$$
\begin{aligned}
& \|T(u)-T(v)\|_{B}=\sup _{t \in R}|T(u)(t)-T(v)(t)| \\
& =\sup _{t \in R} \int_{-\infty}^{t} g(s) \\
& \times \mid \sum_{i=1}^{m} b_{i}(s) \int_{0}^{+\infty} \frac{k_{i}(r)}{1+u^{n}(s-r)} d r \\
& -H(s, u(s-\sigma(s))) \\
& -\sum_{i=1}^{m} b_{i}(s) \int_{0}^{+\infty} \frac{k_{i}(r)}{1+v^{n}(s-r)} d r \\
& -H(s, v(s-\sigma(s))) \mid d s \\
& \leq \sup _{t \in R} \int_{-\infty}^{t} g(s) \\
& \times\left[\sum_{i=1}^{m} b_{i}(s) \int_{0}^{+\infty} \mid \frac{k_{i}(r)}{1+u^{n}(s-r)}\right. \\
& -\frac{k_{i}(r)}{1+v^{n}(s-r)} \mid d r \\
& +|H(s, u(s-\sigma(s)))-H(s, v(s-\sigma(s)))|]
\end{aligned}
$$

$$
\begin{gathered}
\leq \sup _{t \in R} \int_{-\infty}^{t} g(s) \\
\times\left[\sum_{i=1}^{m} b_{i}(s) n\|u-v\| \int_{0}^{+\infty} k_{i}(r) d r\right. \\
\left.+L_{H}\|u-v\|\right] d s \\
\leq \sup _{t \in R} \int_{-\infty}^{t} g(s)\left[n \sum_{i=1}^{m} b_{i}(s)+L_{H}\right] d s\|u-v\| \\
\leq \frac{n \sum_{i=1}^{m} b_{i}^{+}+L_{H}}{a^{-}}\|u-v\| .
\end{gathered}
$$

According to $L_{H}+n \sum_{i=1}^{m} b_{i}^{+} \leq a^{-}$and (23), we know that $T$ is a contraction on $B^{*}$. Thus, $T$ has a unique fixed point $\varphi^{*} \in B^{*}$ such that $T \varphi^{*}=\varphi^{*}$. By (14), $\varphi^{*}$ satisfies (2); then (2) exists as a unique positive almost periodic solution $\varphi^{*}$ in $B^{*}$. The proof of Theorem 8 is complete.

Theorem 9. Assume that (H1)-(H4) and $L_{H}+n \sum_{i=1}^{m} b_{i}^{+} \leq a^{-}$ hold. Let $x^{*}(t)$ be the positive almost periodic solution of (2) in the region $B^{*}$. Then the solution $x\left(t ; t_{0}, \varphi\right)$ of $(2)$ with $\varphi \in C^{0}$ converges exponentially to $x^{*}(t)$ as $t \rightarrow+\infty$.

Proof. Let $x(t)=x\left(t ; t_{0}, \varphi\right)$ and $y(t)=x(t)-x^{*}(t)$, where $t \in\left[t_{0}-r,+\infty\right)$. So we have

$$
\begin{aligned}
y^{\prime}(t)= & -a(t) y(t) \\
& +\sum_{i=1}^{m} b_{i}(t) \int_{0}^{+\infty} k_{i}(r)\left[\frac{1}{1+x^{n}(t-r)}\right. \\
& \left.-\frac{1}{1+x^{* n}(t-r)}\right] d r \\
& -\left[H(t, x(t-\sigma(t)))-H\left(t, x^{*}(t-\sigma(t))\right)\right] .
\end{aligned}
$$

According to Lemma 7, we get that $x(t)$ is positive and bounded on $\left[t_{0},+\infty\right)$ and $M_{2}<x(t)<M_{1}$ for all $t \epsilon$ $\left[t_{0}-r,+\infty\right)$.

Define a continuous function $\Gamma(u)$ by setting

$$
\begin{aligned}
\Gamma(u)= & -\left(a^{-}-u\right) \\
& +n \sum_{i=1}^{m} b_{i}^{+} \int_{0}^{+\infty} k_{i}(t) e^{u t} d t+L_{H} e^{u r}, \\
u & \in[0,1] .
\end{aligned}
$$

Then, we have

$$
\Gamma(0)=-a^{-}+n \sum_{i=1}^{m} b_{i}^{+}+L_{H}<0
$$


which implies that there exists a constant $\lambda \in(0,1]$ such that

$$
\begin{aligned}
\Gamma(\lambda)= & -\left(a^{-}-\lambda\right) \\
& +n \sum_{i=1}^{m} b_{i}^{+} \int_{0}^{+\infty} k_{i}(t) e^{\lambda t} d t+L_{H} e^{\lambda r}<0 .
\end{aligned}
$$

We consider the Lyapunov function

$$
V(t)=|y(t)| e^{\lambda t} .
$$

Calculating the upper right derivative of $V(t)$ along the solution $y(t)$ of $(24)$, we obtain that

$$
\begin{aligned}
D^{+}(V(t)) & \\
\leq & -a(t)|y(t)| e^{\lambda t}+\lambda|y(t)| e^{\lambda t} \\
& +e^{\lambda t} \sum_{i=1}^{m} b_{i}(t) \\
& \times\left[\int_{0}^{+\infty} k_{i}(s)\left|\frac{1}{1+x^{n}(t-s)}-\frac{1}{1+x^{* n}(t-s)}\right| d s\right] \\
& +\left|H(t, x(t-\sigma(t)))-H\left(t, x^{*}(t-\sigma(t))\right)\right| e^{\lambda t} \\
= & {[(\lambda-a(t))|y(t)|] } \\
& +e^{\lambda t} \sum_{i=1}^{m} b_{i}(t) \\
& \times \int_{0}^{+\infty} k_{i}(s)\left|\frac{1}{1+x^{n}(t-s)}-\frac{1}{1+x^{* n}(t-s)}\right| d s \\
+\mid & (t, x(t-\sigma(t)))-H\left(t, x^{*}(t-\sigma(t))\right) \mid e^{\lambda t},
\end{aligned}
$$

We claim that

$$
\begin{aligned}
& V(t)=|y(t)| e^{\lambda t}<e^{\lambda t_{0}} \\
& \times\left(\max _{t \in\left[-r, t_{0}\right]}\left|\varphi(t)-x^{*}(t)\right|+M_{1}\right):=M_{3}, \\
& \forall t>t_{0} .
\end{aligned}
$$

Contrarily, there must exist $t_{*}>t_{0}$ such that

$$
V\left(t_{*}\right)=M_{3}, \quad V(t)<M_{3}, \quad \forall t \in\left[-r, t_{*}\right),
$$

which implies that

$$
V\left(t_{*}\right)-M_{3}=0, \quad V(t)-M_{3}<0, \quad \forall t \in\left[-r, t_{*}\right) .
$$

Because $x(t) \geq 0$ and $x^{*}(t) \geq 0$ for $t \geq-r$.
By (29) and (32), we have

$$
\begin{aligned}
0 \leq & D^{+}\left(V\left(t_{*}\right)-M_{3}\right)=D^{+}\left(V\left(t^{*}\right)\right) \\
\leq & \left(\lambda-a\left(t_{*}\right)\right)\left|y\left(t_{*}\right)\right| e^{\lambda t_{*}} \\
& +e^{\lambda t_{*}} \sum_{i=1}^{m} b_{i}\left(t_{*}\right) \\
& \times \int_{0}^{+\infty} k_{i}(t)\left|\frac{1}{1+x^{n}\left(t_{*}-t\right)}\right| \frac{1}{1+x^{* n}\left(t_{*}-t\right)} \mid d t \\
& +\left|H\left(t_{*}, x\left(t_{*}-\sigma\left(t_{*}\right)\right)\right)-H\left(t_{*}, x^{*}\left(t-\sigma\left(t_{*}\right)\right)\right)\right| e^{\lambda t_{*}} \\
\leq & \left(\lambda-a\left(t_{*}\right)\right)\left|y\left(t_{*}\right)\right| e^{\lambda t_{*}} \\
& +\sum_{i=1}^{m} n b_{i}\left(t_{*}\right) \int_{0}^{+\infty} e^{\lambda t_{*}} k_{i}(t)\left|y\left(t_{*}-t\right)\right| d t \\
& +L_{H} y\left(t_{*}-\sigma\left(t_{*}\right)\right) e^{\lambda t_{*}} \\
\leq & \left(\lambda-a\left(t_{*}\right)\right)\left|y\left(t_{*}\right)\right| e^{\lambda t_{*}} \\
& +\sum_{i=1}^{m} n b_{i}\left(t_{*}\right) \int_{0}^{+\infty} k_{i}(t) e^{\lambda\left(t_{*}-t\right)}\left|y\left(t_{*}-t\right)\right| e^{\lambda t} d t \\
& +L_{H} y\left(t_{*}-\sigma\left(t_{*}\right)\right) e^{\lambda\left(t_{*}-\sigma\left(t_{*}\right)\right)} e^{\lambda \sigma\left(t_{*}\right)} \\
\leq & {\left[\left(\lambda-a^{-}\right)+n \sum_{i=1}^{m} b_{i}^{+} \int_{0}^{+\infty} k_{i}(t) e^{\lambda t} d t+L_{H} e^{\lambda r}\right] M_{3 .} }
\end{aligned}
$$

Obviously, (33) contradicts with (25). Hence, (28) holds. It follows that $|y(t)|<M_{3} e^{-\lambda t}$, for all $t>t_{0}$. Thus, the proof is now completed.

Remark 10. Obviously, we consider system (2) with the nonlinear harvesting term and infinite time delays of (2), and the results of the references therein cannot be applied to prove the existence and exponential convergence of solutions of (2).

\section{An Example}

In this section, we give an example to illustrate the results obtained in the previous sections.

Example 11. Consider the following Hematopoiesis model with infinite delays and nonlinear harvesting term:

$$
\begin{aligned}
x^{\prime}(t)= & -\left(30+\cos ^{2} t\right) x(t) \\
& +(0.003+0.002|\sin \sqrt{2} t|) \int_{0}^{+\infty} \frac{2 e^{-2 s}}{1+x^{4}(t-s)} d s
\end{aligned}
$$




$$
\begin{aligned}
& +(0.005+0.003|\cos \sqrt{3} t|) \int_{0}^{+\infty} \frac{3 e^{-3 s}}{1+x^{4}(t-s)} d s \\
& -0.001|\sin t+\cos \sqrt{5} t| \frac{x^{3}(t-|\sin \sqrt{7} t|)}{1+x^{2}(t-|\sin \sqrt{7} t|)}
\end{aligned}
$$

We know that

$$
\begin{gathered}
\alpha^{-}=30, \quad \alpha^{+}=31, \quad b_{1}^{-}=0.003, \quad b_{1}^{+}=0.005, \\
n=4, \quad k_{1}(t)=2 e^{-2 t}, \quad k_{2}(t)=3 e^{-3 t}, \\
H(t, x)=0.001|\sin t+\cos \sqrt{5} t| \frac{x^{3}}{1+x^{2}}, \\
\sigma(t)=t-|\sin \sqrt{7} t|, \quad t \in R .
\end{gathered}
$$

It is not difficult to obtain that $H(t, x)$ satisfies Lipschitz condition and take $L_{H}=0.002$. It follows from Theorem 9 that Hematopoiesis model (34) has a unique positive almost periodic solution $\tilde{x}(t)$. Moreover, if $\varphi \in C^{0}$, then the solutions $x\left(t ; t_{0}, \varphi\right)$ of (34) converge exponentially to $\tilde{x}(t)$ as $t \rightarrow+\infty$.

Remark 12. It is obvious that the delays in (34) are infinite time delays and a nonlinear harvesting term. This implies that the results of this paper are new and they complement previously known results [14-17].

\section{Acknowledgments}

The authors would like to express sincere appreciation to the editor and referee for their valuable comments and good suggestions which have led to an improvement in the presentation of the paper. Research is supported by the National Natural Science Foundation of China (11071001) and Anhui Province Natural Science Foundation (1208085MA13).

\section{References}

[1] Y. S. Chen, "The existence of periodic solutions for a class of neutral differential-difference equations," Australian Mathematical Society B, vol. 33, no. 4, pp. 508-516, 1992.

[2] Y. Qu, M. Y. Li, and J. Wei, "Bifurcation analysis in a neutral differential equation," Journal of Mathematical Analysis and Applications, vol. 378, no. 2, pp. 387-402, 2011.

[3] Z. Li and F. Chen, "Almost periodic solutions of a discrete almost periodic logistic equation," Mathematical and Computer Modelling, vol. 50, no. 1-2, pp. 254-259, 2009.

[4] Y. Wang and Y.-H. Xia, "The existence of almost periodic solutions of a certain nonlinear system," Communications in Nonlinear Science and Numerical Simulation, vol. 16, no. 2, pp. 1060-1072, 2011.

[5] S. Abbas and D. Bahuguna, "Almost periodic solutions of neutral functional differential equations," Computers \& Mathematics with Applications, vol. 55, no. 11, pp. 2593-2601, 2008.

[6] X. Chen and F. Lin, "Almost periodic solutions of neutral functional differential equations," Nonlinear Analysis: Real World Applications, vol. 11, no. 2, pp. 1182-1189, 2010.
[7] C. Y. He, Almost Periodic Differential Equations, Higher Education Publishing House, Beijing, China, 1992, (Chinese).

[8] K. Gopalsamy and S. I. Trofimchuk, "Almost periodic solutions of Lasota-Wazewska-type delay differential equation," Journal of Mathematical Analysis and Applications, vol. 237, no. 1, pp. 106127, 1999.

[9] A. M. Fink, Almost Periodic Differential Equations, vol. 377 of Lecture Notes in Mathematics, Springer, Berlin, Germany, 1974.

[10] E. Braverman and S. H. Saker, "Permanence, oscillation and attractivity of the discrete hematopoiesis model with variable coefficients," Nonlinear Analysis: Theory, Methods \& Applications, vol. 67, no. 10, pp. 2955-2965, 2007.

[11] G. Liu, J. Yan, and F. Zhang, "Existence and global attractivity of unique positive periodic solution for a model of hematopoiesis," Journal of Mathematical Analysis and Applications, vol. 334, no. 1, pp. 157-171, 2007.

[12] M. Adimy and F. Crauste, "Mathematical model of hematopoiesis dynamics with growth factor-dependent apoptosis and proliferation regulations," Mathematical and Computer Modelling, vol. 49, no. 11-12, pp. 2128-2137, 2009.

[13] S. H. Saker and J. O. Alzabut, "On the impulsive delay hematopoiesis model with periodic coefficients," The Rocky Mountain Journal of Mathematics, vol. 39, no. 5, pp. 1657-1688, 2009.

[14] J. O. Alzabut, J. J. Nieto, and G. Tr. Stamov, "Existence and exponential stability of positive almost periodic solutions for a model of hematopoiesis," Boundary Value Problems, vol. 2009, Article ID 127510, 10 pages, 2009.

[15] X. Wang and Z. Li, "Dynamics for a class of general hematopoiesis model with periodic coefficients," Applied Mathematics and Computation, vol. 186, no. 1, pp. 460-468, 2007.

[16] X. Wang and H. Zhang, "A new approach to the existence, nonexistence and uniqueness of positive almost periodic solution for a model of hematopoiesis," Nonlinear Analysis: Real World Applications, vol. 11, no. 1, pp. 60-66, 2010.

[17] S. H. Saker, "Oscillation and global attractivity in hematopoiesis model with delay time," Applied Mathematics and Computation, vol. 136, no. 2-3, pp. 241-250, 2003.

[18] M. C. Mackey and L. Glass, "Oscillatios and chaos in physiological control system,” Sciences, vol. 197, pp. 287-289, 1977.

[19] D. Jiang, D. O'Regan, R. P. Agarwal, and X. Xu, "On the number of positive periodic solutions of functional differential equations and population models," Mathematical Models \& Methods in Applied Sciences, vol. 15, no. 4, pp. 555-573, 2005.

[20] H. Zhou, Z. Zhou, and Q. Wang, "Positive almost periodic solution for a class of Lasota-Wazewska model with infinite delays," Applied Mathematics and Computation, vol. 218, no. 8, pp. 4501-4506, 2011.

[21] H. Zhou, J. Wang, and Z. Zhou, "Positive almost periodic solution for impulsive Nicholson's blowflies model with multiple linear harvesting terms," Mathematical Methods in the Applied Sciences, vol. 36, no. 4, pp. 456-461, 2013.

[22] F. Long and M. Q. Yang, "Positive periodic solutions of delayed Nicholsons blowies model with a linear harvesting term," Electronic Journal of Qualitative Theory of Differential Equations, vol. 41, pp. 1-11, 2011.

[23] L. Wang, "Almost periodic solution for Nicholson's blowflies model with patch structure and linear harvesting terms," Applied Mathematical Modelling, vol. 37, no. 4, pp. 2153-2165, 2013.

[24] J. Hale, Theory of Functional Differential Equations, vol. 3, Springer, New York, NY, USA, 2nd edition, 1977, Applied Mathematical Sciences. 


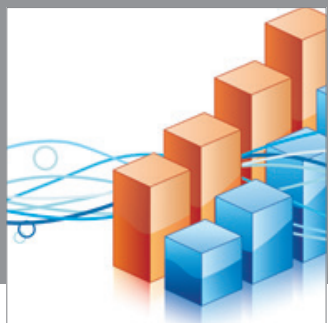

Advances in

Operations Research

mansans

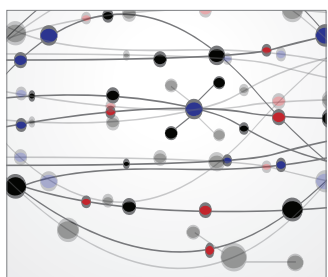

The Scientific World Journal
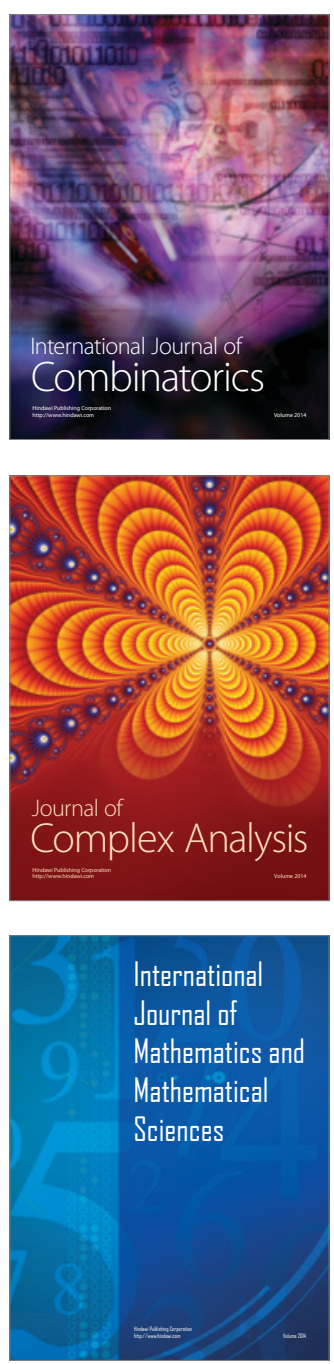
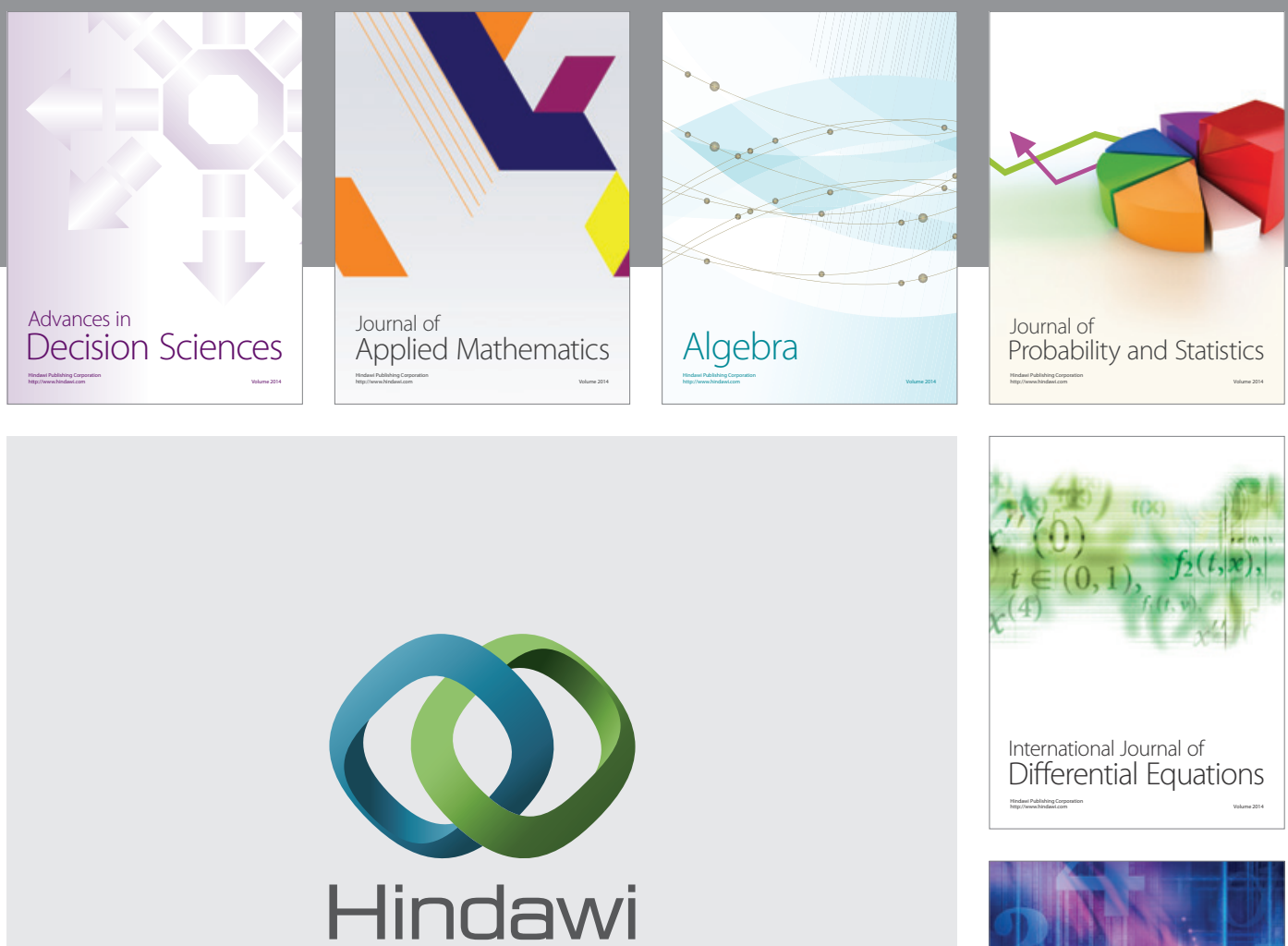

Submit your manuscripts at http://www.hindawi.com
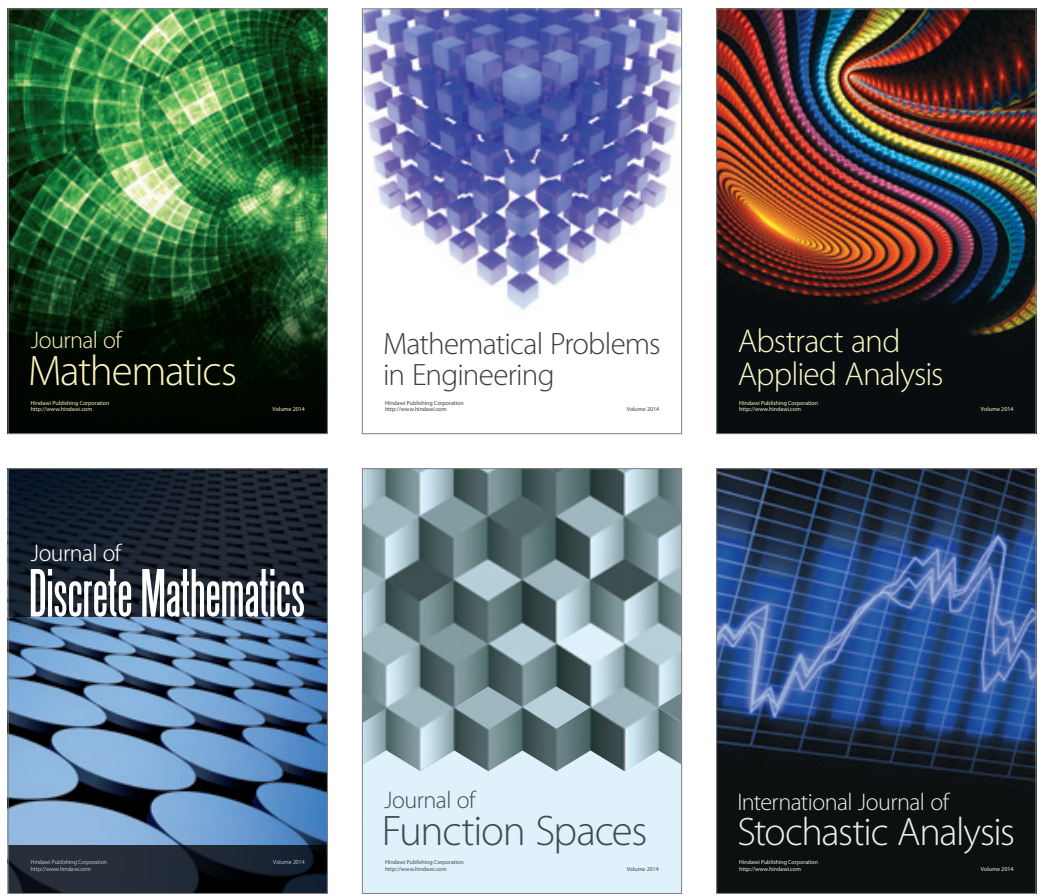

Journal of

Function Spaces

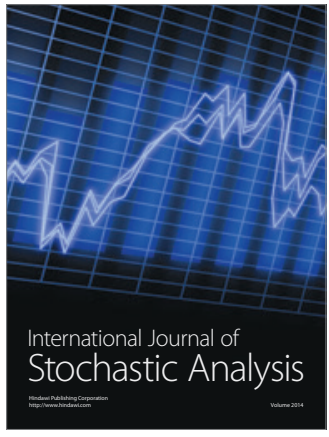

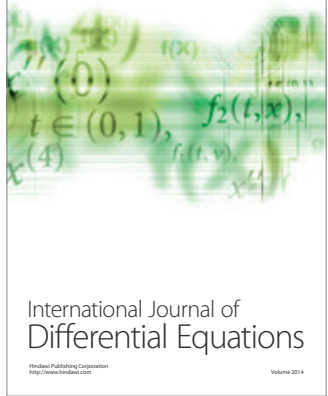
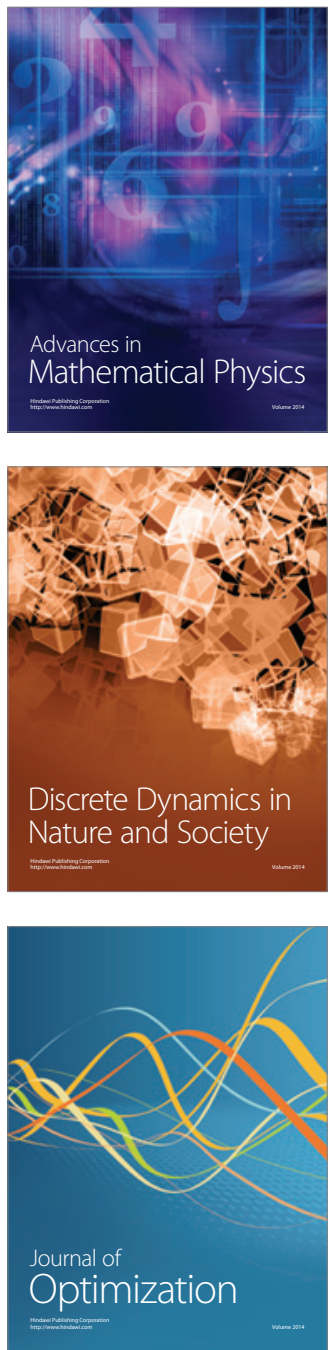